\title{
Intégration du Traitement Spécial et Différencié dans le cadre des Accords de Partenariat Economique entre l'Union Européenne et les Etats d'Afrique, Caraïbes et Pacifique.
}

\author{
Calliste NIZANA ${ }^{1-2}$ \\ ${ }^{1}$ (Maître-Assistant d'Enseignement à la Faculté de Droit/ Université du Burundi, Burundi) \\ ${ }_{2}^{2}$ (Doctorant au Centre des Etudes Doctorales en Droit et Economie-Agdal/ Université Mohamed V, Maroc)
}

\begin{abstract}
Therevision of the cooperation between the European Union (EU) and the African, Caribbean and Pacific (ACP) States under the Cotonou Agreement signed in 2000, provided for the establishment of Economic Partnership Agreements (EPAs) compatible with Article XXIV of the General Agreement on Tariffs and Trade (GATT). This reform of the trade regime of the Cotonou Agreement raised a controversy between the EU and the ACP States on the possibility of incorporating provisions on Special and Differential Treatment (SDT), a principle recognized by the WTO, in EPAs. Given that Article XXIV of the GATT does not mention explicitly the SDT principle, its interpretation and the negotiations between the EU and ACP states have resulted in integration of SDT principle in the context of the Cotonou Agreement and EPAs. The main provisions included in the EPAs are those relating to trade capacity building in the ACP States and those relating to asymmetrical trade liberalization between the EU and the ACP countries that is to say the flexibilities in terms of commitments and longer transition periods for ACP States.
\end{abstract}

Keywords -African, Caribbean and Pacific States, Asymmetric liberalization, Cotonou Agreement, Economic Partnership Agreements, European Union, Special and Differential Treatment, World Trade Organization.

Résumé: La révision de la coopération entre l'Union Européenne (UE) et les Etats d'Afrique, Caraïbes et Pacifique (ACP), dans le cadre de l'Accord de Cotonou signé en 2000, prévoyait la mise en place d'Accords de Partenariat Economique (APE) compatibles avec l'Article XXIV de l'Accord Général sur les Tarifs douaniers et le Commerce (ou GATT) de l'Organisation Mondiale du Commerce (OMC). Cette réforme du régime commercial de l'Accord de Cotonou a soulevé une controverse entre l'UE et les Etats ACP concernant la possibilité d'intégrer des dispositions relatives au Traitement Spécial et Différencié (TSD), principe reconnu à l'OMC, dans le cadre des APE. Vu que l'Article XXIV du GATT ne traite pas expressément du TSD, son interprétation ainsi que les négociations entre l'UE et les Etats ACP, ont abouti à une intégration du principe du TSD dans le cadre de l'Accord de Cotonou et des APE. Les principales dispositions intégrées dans le cadre des APE sont celles relatives au renforcement des capacités commerciales des Etats ACP et celles relatives à une libéralisation asymétrique des échanges entre l'UE et les Etats ACP c'est-à-dire des flexibilités en matière d'engagements et des périodes de transition plus longues en faveur des Etats ACP.

Mots clés : Accord de Cotonou, Accords de Partenariat économique, Etats ACP (Afrique, Caraïbes et Pacifique), Libéralisation asymétrique, Organisation Mondiale du Commerce, Traitement Spécial et Différencié, Union Européenne.

\section{INTRODUCTION}

La remise en cause des préférences commerciales unilatérales en faveur des Etats d'Afrique, Caraïbes et Pacifique (ACP) dans le cadre des conventions successives de Loméa entrainé une exigence de compatibilité du régime commercial de la coopération entre l'Union Européenne (UE) et les Etats ACP avec les règles de l'Organisation Mondiale du Commerce (OMC).Pour se conformer aux exigences de l'OMC, l'UE et les Etats ACP ont décidé de conclure des Accords de Partenariat Economique (APE) compatibles avec l'article XXIV de l'Accord Général sur les Tarifs douaniers et le Commerce (ou GATT selon l'abréviation en anglais pour "General Agreement on Tariffs and Trade"). Ce choix est étonnant puisque cette solution n'est pas la plus favorable aux Etats ACP d'autant plus que d'autres solutions alternatives avaient été proposées [1]. De plus, en vertu de l'Article XXIV du GATT, les APE doivent être des accords de libre-échange entre l'UE et les Etats ACP alors que les Etats de l'UE et les Etats ACP ne sont pas à des niveaux de développement comparables. En effet, les Etats européens sont considérés comme des pays développés ou industrialisés alors que la plupart des Etats ACP sont considérés comme étant des Pays les Moins Avancés (PMA)[2].

Sachant que l'Article XXIV du GATT ne traite pas expressément du Traitement Spécial et Différencié (TSD) [3], le début des négociations des APE a été marqué par une controverse entre les Etats ACP et l'UE sur 
la possibilité d'intégrer des dispositions relatives au TSD dans le cadre des APE. La question qui se posait était de savoir si les Etats ACP, en tant que pays en développement ou PMA, avaient le droit à un TSD tout en respectant les exigences de l'Article XXIV du GATT relatives à l'établissement d'Accords Commerciaux Régionaux (ACR). Face aux lacunes du cadre juridique en la matière, certaines analyses plaident pour l'application du principe de TSD aux APE. Cet article se propose alors de passer en revue les arguments en faveur d'une intégration du TSD dans le cadre des APE et les dispositions relatives au TSD finalement intégrées dans le cadre des APE déjà signés. On remarque alors que la pratique au sein de l'OMC est plutôt favorable à une libéralisation asymétrique dans le cadre des ACR dits mixtes c'est-à-dire ceux impliquant des pays développés et des pays en développement. Aussi, après avoir manifesté la volonté de prendre en considération les dispositions relatives au TSD, les Etats ACP et l'UE ont intégré certaines flexibilités dans le cadre des APE déjà signés.

\section{INTEGRATION DU TSD DANS LE CADRE DES ACR : LA PRATIQUE A L'OMC FACE AUX AMBIGUÏTES DE L'ARTICLE XXIV DU GATT}

Si l'intégration du TSD dans le cadre des ACR soulève un débat, c'est principalement parce qu'il n'y a pas de lien juridique entre l'Article XXIV du GATT et le TSD. En effet, parmi les différentes dispositions reconnues comme faisant référence au principe du TSD dans les Accords et Décisions de l'OMC, aucune ne se retrouve dans le cadre de l'Article XXIV du GATT. Face aux lacunes juridiques, la possibilité d'une libéralisation asymétrique dans le cadre d'un ACR tient à l'interprétation que l'on fait de l'Article XXIV du GATT.

\section{Absence de lien juridique entre l'Article XXIV du GATT et le TSD}

L'Article XXIV du GATT ne traite pas expressément des questions relatives au développement et ne contient donc aucune disposition relative au TSD. Cette absence de lien juridique entre l'Article XXIV du GATT et le TSD s'explique par deux raisons principales. La première raison tient au fait que "l'article XXIV du GATT précède de plusieurs décennies l'institutionnalisation du TSD» [4] et lorsque des dispositions relatives au TSD ont été introduites dans les différents Accords de l'OMC, l'Article XXIV du GATT n'a pas été modifié en ce sens. En effet, la Partie IV du GATT qui traite du «commerce et développement », qui a été ajoutée au GATT de 1947 par un protocole d'amendement en 1964, est entrée formellement en vigueur le 27 Juin 1966. La Clause d'habilitation [5], qui est l'un des fondements juridiques du TSD a été adoptée en 1979. De plus, le GATT de 1947 ne faisait pas de distinction entre pays développés et pays en développement parmi les «Parties Contractantes » ce qui, par conséquent, bloque un traitement préférentiel envers telle ou telle Partie Contractante sur la base du niveau de développement dans le cadre des ACR.

La seconde raison tient au fait que les premiers ACR étaient généralement conclus entre des pays à des niveaux de développement comparables. Il n'existait pas encore d'ACR qu'on qualifierait aujourd'hui d'accords commerciaux nord-sud. Par conséquent les pays qui signaient des ACR à l'époque n'étaient pas motivés par une possibilité d'obtenir un traitement préférentiel.

\section{Une interprétation souple de l'Article XXIV du GATT et une pratique des Membres de l'OMC favorables à une libéralisation asymétrique.}

Les défenseurs d'une libéralisation asymétrique entre pays développés et pays en développement dans le cadre des ACR qualifiés de «Nord-Sud » ou de «mixtes » insistent sur une interprétation souple des deux notions controversées de l'Article XXIV du GATT à savoir «l'essentiel des échanges commerciaux » et le «délai raisonnable ».

\subsection{Interprétation de «l'essentiel des échanges » et libéralisation asymétrique}

L'Article XXIV du GATT dispose que dans le cadre d'une Zone de Libre-échange, les droits de douane doivent être éliminés pour «l'essentiel des échanges commerciaux portant sur les produits originaires des territoires constitutifs de la zone de libre-échange » [6], sans pour autant donner une définition de "l'essentiel des échanges ». Par conséquent, aucune définition concernant «l'essentiel des échanges commerciaux » n'est consensuelle au sein de l'OMC. Le seul consensus sur l'Article XXIV.8 du GATT, c'est qu'il n'y a pas de consensus sur son interprétation, et donc sur les modalités de son application [7]. Même l'Organe d'appel de l'OMC n'a pas pu éclairer les Membres puisqu'il a déclaré que « l'essentiel des échanges commerciaux » n'est pas la même chose que la totalité des échanges commerciaux, et qu'il s'agit de quelque chose de beaucoup plus 
important que simplement une certaine partie des échanges et que cela offre « une certaine souplesse » aux membres constitutifs d'une union douanière dans la libéralisation de leur commerce interne.

La flexibilité dans la libéralisation dans le cadre de l'Article XXIV.8, a donné lieu à deux approches, l'une qualitative et l'autre quantitative. Dans le cadre d'une approche quantitative, on préconise la définition d'un repère statistique en volume. Ce repère est ainsi exprimé en termes de pourcentage des échanges à libéraliser. Les tenants de l'approche qualitative quant à eux, stipulent qu'il faut prendre en compte les produits visés par la libéralisation et recommandent qu'aucun secteur important ne soit exclu de la libéralisation des échanges.

L'UE adopte généralement l'interprétation quantitative et détermine que «l'essentiel des échanges » signifie une libéralisation d'environ 80 à $90 \%$ des échanges. Les Etats ACP ont, quant à eux, proposé une interprétation qui permettrait d'introduire une asymétrie dans la libéralisation commerciale. En effet, si la pratique des Membres de l'OMC laisse penser que dans un ACR environ $80 \%$ des échanges doivent être libéralisés; la question est de savoir si les $80 \%$ constituent un seuil ou une moyenne des échanges qui doivent être libéralisés. S'il s'agit d'un seuil, les Etats ACP et l'UE doivent libéraliser leurs marchés au moins à hauteur de $80 \%$ chacun pour que les APE soient compatibles avec les règles de l'OMC. Les tenants de cette approche se basent sur le fait que l'Article XXIV du GATT stipule que l'essentiel des échanges commerciaux qui doit être libéralisé porte sur les «produits originaires des territoires constitutifs de la Zone de libre échange ». Ils en déduisent donc que chaque territoire constitutif d'une Zone de libre-échange doit libéraliser au moins $80 \%$ de ses échanges.

Cependant, l'Article XXIV du GATT n'indique pas non plus que la part des échanges libéralisée doit être équitablement répartie entre les Parties ce qui ouvre la voie à l'interprétation qui estime que les $80 \%$ doivent être considérés comme une moyenne. Dans ce cas l'UE pourrait libéraliser 100\% de son marché et les Etats ACP pourraient ouvrir leurs marchés à hauteur de $60 \%$.

\subsection{Le « délai raisonnable » et la détermination de la période de transition}

L'Article XXIV du GATT reste aussi vague concernant le délai nécessaire pour libéraliser les échanges commerciaux dans le cadre d'une Zone de Libre-Echange et parle d'un « délai raisonnable ». Le Mémorandum d'Accord sur l'interprétation de l'Article XXIV du GATT estime que ce " "délai raisonnable" mentionné au paragraphe 5 c) de l'article XXIV ne devrait dépasser 10 ans que dans des cas exceptionnels » sans toutefois préciser ce qu'il faut comprendre par "cas exceptionnels".

Cependant, la pratique à l'OMC ouvre la voie à une libéralisation asymétrique dans le cadre des APE. En effet, l'analyse des ACR notifiés à l'OMC au titre de l'Article XXIV du GATT montre que, dans certains cas, les délais de mise en œuvre dépassent largement 10 ans (Tableau 1) et « ces cas deviennent la règle plutôt que l'exception» [8]. Il est apparu alors que la grande majorité des Zones de Libre-Echange entre pays développés et pays en développement sont asymétriques et qu'un délai systématique de 20 ans n'est en rien exceptionnel.

Tableau 1 : Exemples d'ACR ayant dépassé dix ans de mise en œuvre.

\begin{tabular}{|l|c|}
\hline \multicolumn{1}{|c|}{ ACR } & Délai de mise en ouvre \\
\hline UE-Maroc & 12 ans \\
\hline Japon-Philippines & 16 ans \\
\hline Australie-Etats-Unis & 18 ans \\
\hline Canada-Chili & 18 ans \\
\hline Thaillande-Australie & 20 ans \\
\hline Thaillande-Nouvelle Zélande & 20 ans \\
\hline USA-Maroc & 24 ans \\
\hline
\end{tabular}

Source : Réalisation de l'auteur

Si des pays développés comme l'Australie et les Etats-Unis disposent d'une période de 18 ans pour mettre en œuvre leur accord de libre-échange, il apparait alors légitime de considérer que la situation économique des Etats ACP, qui sont en majorité des PMA, constitue un "cas exceptionnel" pour justifier des périodes de transition plus longues dans la mise en œuvre des APE.

Les négociations des APE entre l'UE et les Etats ACP ont abouti à une libéralisation asymétrique puisqu'elles ont tenu compte des différentes interprétations de l'Article XXIV du GATT et de la pratique de certains Membres de l'OMC.

\section{LES PRINCIPALES DISPOSITIONS RELATIVES AU TSD INTEGREES DANS LE CADRE DES APE}

L'Accord de Cotonou signé en 2000 aborde globalement la coopération entre les Etats ACP et l'UE alors que l'aspect commercial de la coopération est traité dans le cadre des APE. Les APE sont des accords commerciaux entre l'UE d'une part et sept groupements régionaux au sein des Etats ACP d'autre part, chaque groupement 
régional négociant son propre APE avec l'UE. Ces groupements régionaux sont le CARIFORUM qui regroupe les Etats ACP des Caraïbes, le groupement régional « Afrique Centrale », la région « Pacifique » qui regroupe les Etats ACP du Pacifique, la Communauté d'Afrique de l'Est, le groupement régional « Afrique de l'Ouest » qui englobe les Etats ACP de l'Afrique de l'Ouest, le groupement régional «AFOA » (pour Afrique Orientale et Australe) et la région SADC (pour SouthernAfricanDevelopmentCommunity).

La question relative au TSD était déjà évoquée dans le cadre de l'Accord de Cotonou. Les Etats ACP et l'UE reconnaissent, en effet, la nécessité d'intégrer des dispositions relatives au TSD dans le cadre des APE. Ainsi, l'Accord de Cotonou stipule que le régime commercial de la coopération doit être mis en conformité avec les règles de l'OMC « ... y compris un traitement spécial et différencié tenant compte des intérêts mutuels des parties et de leurs niveaux respectifs de développement. »[9]. Dans l'analyse des dispositions relatives au TSD inscrites dans le cadre des APE, on adopte la même classification que celle faite par l'OMC. En effet, si les Etats $\mathrm{ACP}$ ont réussi à faire intégrer le TSD dans le cadre des APE, celui-ci doit se conformer à ce qui est juridiquement autorisé par les règles de l'OMC. Cependant, dans le cadre de cet article, on s'intéresse uniquement à trois catégories qui sont directement concernées par la controverse suscité par l'Article XXIV du GATT. Il s'agit des dispositions relatives à l'accroissement des possibilités commerciales des Etats ACP, aux flexibilités des engagements et aux périodes transitoires.

\section{Accroissement des possibilités commerciales des Etats ACP : Accompagnement des préférences commerciales par une aide pour le commerce}

Selon 1'OMC, les dispositions relatives au TSD ayant pour objectif d'accroitre les possibilités commerciales des pays en développement et PMA sont celles concernant les actions que les Membres doivent mener pour accroître les possibilités commerciales des pays en développement.Ces actions doivent être entreprises dans des domaines qui intéressent particulièrement les pays en développement. Dans le cadre de l'accord de Cotonou, l'UE et les Etats ACP reconnaissent la nécessité d'accorder une attention particulière "à la vulnérabilité de nombreux États ACP résultant de leur dépendance à l'égard des produits de base ou de quelques produits clés, notamment des produits agro-industriels à valeur ajoutée, ainsi qu'à l'érosion des préférences » [10].

Sachant que les négociations des APE ne sont pas encore terminées sauf pour l'APE entre l'UE et la région CARIFORUM, les différents APE ne contiennent que des dispositions relatives au commerce des marchandises. Par conséquent, dans le cadre des APE qui ont été déjà signés qu'ils soient complets ou intérimaires, les dispositions ayant pour objectif d'accroitre les possibilités commerciales des Etats ACP sont celles qui leur octroient un accès préférentiel au marché européen. Ainsi, l'UE octroie aux Etats ACP qui ont accepté de signer des APE, un accès en franchise de droits de douane et sans contingents au marché européen.

$\mathrm{Si}$ les préférences commerciales de Lomé ont été remises en cause, ce n'est pas parce qu'elles étaient unilatérales mais parce qu'elles étaient discriminatoires à l'égard des autres Membres de l'OMC en particulier les autres pays en développement qui ne font pas partie du Groupe ACP. L'Article XXIV du GATT, qui est une exception au principe de non-discrimination, admet une discrimination dans le cadre des ACR (à l'égard des pays qui ne font pas partie de l'ACR), à condition que les préférences commerciales soient réciproques entre les membres de l'ACR. Par conséquent, l'UE peut continuer à accorder des préférences commerciales aux Etats $\mathrm{ACP}$ en leur garantissant un accès au marché européen en franchise de droits de douane et sans contingents sauf que désormais les Etats ACP doivent aussi ouvrir leurs marchés aux produits en provenance de l'UE.

Le régime commercial des APE est jugé plus préférentiel par rapport à d'autres régimes commerciaux de l'UE notamment le Système de Préférences Généralisées. L'accès au marché européen dans des conditions préférentielles et plus favorables confère ainsi un avantage considérable aux Etats ACP par rapport à d'autres pays. En effet, les autres pays en développement qui sont parfois des concurrents des Etats ACP sur le marché européen, ont un accès moins privilégié. Les PMA non ACP sont soumis au régime dit «Tout sauf les armes »et accèdent au marché européen en franchise de droits de douanes et sans contingents, mais ce régime commercial est un régime unilatéralement conçu par l'UE et est donc susceptible d'être modifié de façon unilatéral. Il y a donc une certaine incertitude pour les exportations de ces PMA sur le marché européen. Les pays en développement non $\mathrm{ACP}$, qui ne sont pas des PMA accèdent au marché européen sous le régime général du Système de Préférences Généralisées, or celui-ci est jugé moins favorable par rapport aux préférences commerciales octroyées dans le cadre des APE et la liste de ses bénéficiaires est susceptible d'être modifiée unilatéralement par l'UE. Actuellement, l'APE entre le CARIFORUM et l'UE est le seul qui traite des sujets autres que le commerce des marchandises. Il s'agit notamment du commerce des services et l'APE reconnait un traitement préférentiel en faveur des prestataires de services originaires du CARIFORUM en particulier dans le secteur du tourisme. L'accord de Cotonou prévoit aussi de « renforcer les capacités de production, d'offre et de commercialisation des pays ACP, ainsi que leur capacité à attirer les investissements » [11]. Il est ainsi prévu un accompagnement financier pour la mise en œuvre des APE. Cet accompagnement, qui est octroyé sous forme d'aide pour le commerce, est primordial pour les Etats ACP parce qu'il s'est avéré que le fait de bénéficier de préférences commerciales n'est pas suffisant pour leur assurer une intégration effective dans le commerce 
internationald'autant plus que les APE sont des accords de libre-échange entre des partenaires économiquement inégaux. L'accès préférentiel aux marchés doit être accompagné par une aide pour le commerce qui contribue à l'abolition d'obstacles contre lesquels se heurtent particulièrement les pays pauvres faute de capacité humaine, institutionnelle et de production [12]. Cependant, pour profiter de l'aide pour le commerce, les Etats ACP doivent se l'approprier et intégrer le commerce dans leurs stratégies de développement. C'est dans ce contexte que les Etats ACP de l'Afrique de l'Ouest ont négocié avec l'UE pour mettre en place le Programme APE pour le développement (PAPED). Il s'agit d'un cadre de mise en œuvre de l'aide pour le commerce en faveur des Etats ACP pour les aider à profiter pleinement de l'APE qu'ils ont signé avec l'UE. Les autres groupements régionaux au sein du Groupe ACP sont aussi en train de négocier des programmes d'accompagnement des APE. Ces programmes d'accompagnement des APE sont considérés par les Etats ACP comme étant « la dimension développement» des APE; raison pour laquelle ils insistent pour que l'UE puisse leur accorder un accompagnement financier conséquent.

\section{Asymétrie des engagements et périodes transitoires}

Les pays en développement en général et les PMA en particulier réclament souvent des flexibilités en matière d'engagements et des périodes transitoires plus longues dans le cadre des accords commerciaux. Dans le cadre de la libéralisation des échanges, les flexibilités traduisent une certaine asymétrie des engagements, les pays en développement pouvant souscrire à des engagements moins importants que les pays développés. De plus, la mise en œuvre des accords commerciaux peut exiger des ajustements ou des mises à niveau des économies, raison pour laquelle les pays en développement demandent souvent des délais plus longs pour mettre en œuvre les engagements consentis. L'accord de Cotonou stipule ainsi que les négociations des APE tiennent compte du niveau de développement et de l'incidence socio-économique des mesures commerciales sur les pays $\mathrm{ACP}$ et de leur capacité à s'adapter et à ajuster leurs économies au processus de libéralisation. Il ajoute par ailleurs que les négociations seront donc aussi flexibles que possible en ce qui concerne la fixation d'une période de transition d'une durée suffisante, la couverture finale des produits, en tenant compte des secteurs sensibles, et le degré d'asymétrie en termes de calendrier du démantèlement tarifaire, tout en restant conformes aux règles de l'OMC en vigueur à cette date.Les négociations des APE ont permis de définir les flexibilités qui pouvaient s'appliquer aux Etats ACP et les périodes de transition nécessaires pour mettre en œuvre les engagements pris dans le cadre des APE. L'analyse des APE déjà signés et ceux toujours en négociation montre que les degrés de libéralisation et les périodes transitoires varient en fonction des pays et en fonction de la sensibilité des marchandises en provenance de l'UE. Les réductions des droits de douane sur les produits sensibles sont réalisées progressivement et sur de longues périodes de transition. Dans le cadre de l'APE entre l'UE et la région CARIFORUM, qui est un APE complet, le CARIFORUM a consenti de libéraliser son marché progressivement pour les importations en provenance de l'UE sur une période maximale de 25 ans. Cependant, cette période de transition à première vue très longue ne s'applique qu'à quelques produits considérés comme étant sensibles et ne représentant qu'une petite part des importations en provenance de l'UE. Les droits de douane sur la plupart des marchandises seront, en effet, éliminés sur une période de 15 ans sachant que pour certains produits ils ont été éliminés à hauteur de $50 \%$ voire $75 \%$ dès l'entrée en vigueur de l'APE en 2009. La région CARIFORUM a pu exclure certains produits de la libéralisation auxquels elle applique les droits de douane consentis dans le cadre du régime de la Nation la Plus Favorisée (NPF). Une asymétrie existe aussi dans la libéralisation du commerce des services. En effet, alors que l'UE va libéraliser 94\% de son marché des services, les Etats du CARIFORUM libéraliseront $65 \%$ pour les PMA et $75 \%$ pour les autres sauf pour la République Dominicaine qui a consenti de libéraliser $90 \%$ de son marché.Dans la région « Afrique centrale », seul le Cameroun a signé un APE intérimaire et dispose de 15 ans pour libéraliser 80\% des échanges commerciaux en provenance de l'UE. Cependant, la période de 15 ans étant une période maximale, la libéralisation du marché camerounais est progressive. Les produits européens ont été ainsi classés en différentes catégories. Les produits de la première catégorie ont été libéralisés après une période de transition de 5 ans, ceux de la deuxième catégorie après 10 ans et ceux de la troisième catégorie seront complètement libéralisés après une période transitoire de 15 ans. Les produits des catégories suivantes ont été exclus de la libéralisation et restent soumis au régime de la Nation la Plus Favorisée. Il faut cependant souligner qu'il s'agit d'un accord intérimaire vu que les autres pays de l'Afrique centrale ne l'ont pas signé et reste toujours en cours de négociation. L'UE assure que l'accord reste ouvert à tout autre pays de l'Afrique Centrale qui voudrait y adhérer. La Papouasie-Nouvelle Guinée et Fiji sont les seuls à avoir signé un APE intérimaire dans la région « Pacifique ». La Papouasie-Nouvelle Guinée a consenti de libéraliser 88,1\% en valeur des importations en provenance de l'UE, ce qui représente $82.1 \%$ de ses lignes tarifaires. Aucune période de transition n'a été prévue et cette ouverture du marché devrait être effective dès l'entrée en vigueur de l'APE intérimaire. Quant à Fiji, il a consenti à libéraliser $87,2 \%$ en valeur des importations en provenance de l'UE, qui représentent 79,7\% de ses lignes tarifaires, pendant une période de transition de 15 ans.La Communauté d'Afrique de l'Est devrait avoir 15 ans pour libéraliser progressivement 82,6\% des importations en provenance de l'UE en raison de 
$64,4 \%$ après 10 ans, $15,3 \%$ après 15 ans mais les $2,9 \%$ restants constitués de produits sensibles seront libéralisés sur une période de 25 ans. Cependant, les 82,6\% exprimés en valeur des importations en provenance de l'UE représentent 91,3\% des lignes tarifaires de la Communauté d'Afrique de l'Est. En Afrique de l'Ouest, l'APE prévoit une période de transition de 20 ans pour libéraliser $75 \%$ des lignes tarifaires. Les produits en provenance de l'UE ont été classés en quatre catégories A, B, C et D. 75 lignes tarifaires des produits de la catégorie A devraient être libéralisés dès l'entrée en vigueur de l'Accord et le reste après 5 ans. Les produits de la catégorie B sont libéralisés progressivement après 5 ans et sur une période de 10 ans. Les produits de la catégorie $\mathrm{C}$ sont eux libéralisés après 5 ans mais progressivement sur une période de 15 ans ; alors que ceux de la catégorie D sont exclus de la libéralisation. Dans le cadre de l'APE intérimaire entre l'UE et l'Afrique Orientale et Australe, six États (Comores, Madagascar, Maurice, Seychelles, Zambie et Zimbabwe) avaient participé aux négociations avec l'UE mais l'accord a été signé par uniquement quatre pays (Madagascar, Maurice, Seychelles et Zimbabwe) en août 2009, à Maurice. Madagascar, Maurice, les Seychelles et le Zimbabwe ont consenti de libéraliser respectivement $81,96,98$ et $80 \%$ des échanges avec l'UE sur 15 ans dont une période préparatoire de 5 ans.

Tableau 2 : Périodes de transition dans la mise en ouvre des APE

\begin{tabular}{|l|c|}
\hline \multicolumn{1}{|c|}{ APE } & Période de transition \\
\hline UE-CARIFORUM & 25 ans \\
\hline UE-CAE & 25 ans \\
\hline UE-Cameroun & 15 ans \\
\hline UE-SADC & 15 ans \\
\hline UE-Afrique de l'ouest & 20 ans \\
\hline UE-Pacifique (Fiji uniquement) & 15 ans \\
\hline UE-AFOA & 15 ans \\
\hline
\end{tabular}

Source : Réalisation de l'auteur

Dans la région SADC, le Botswana, le Lesotho, le Mozambique, la Namibie, l'Afrique du Sud et le Swaziland ont signé un APE avec l'UE. Plusieurs disparités existent dans cette région. En effet, cinq Etats de la SADC constituent l'Union douanière d'Afrique australe (SouthernAfrican Custom Union (SACU) en anglais). Cette Union douanière a consenti à libéraliser $86,2 \%$ des échanges en provenance de l'UE sur une période de 15 ans sauf pour l'Afrique du Sud pour laquelle cette période de transition est de 10 ans. De plus, alors que les autres pays de la SACU ont un accès en franchise de droits de douane sur le marché européen, celui n'est ouvert qu'à hauteur de 98,7\% pour l'Afrique du Sud. Le Mozambique, qui ne fait pas partie de la SACU, a consenti de libéraliser $74 \%$ de ses échanges en provenance de l'UE sur une période transitoire de 12 ans.

\section{CONCLUSION}

Les Etats ACP et l'UE ont pu intégrer des dispositions relatives au TSD dans le cadre des APE, bien que l'Article XXIV du GATT offre peu de possibilités du fait qu'il ne traite pas expressément du principe du TSD. Les principales dispositions ainsi intégrées sont celles relatives à l'accroissement des possibilités commerciales des Etats ACP en leur accordant l'accès au marché européen en franchise de droits de douane et sans contingents et une aide pour le commerce pour renforcer leurs capacités de production et de commercialisation. Les Etats ACP ont eu la possibilité de souscrire à des engagements moins importants en matière de libéralisation sauf pour l'Afrique du Sud qui devra ouvrir son marché à hauteur de 98,7\%. Ils auront aussi des périodes de transition relativement longues pour mettre en œuvre les APE, les délais de mise en œuvre variant de 10 à 25 ans parfois avec une période préparatoire de 5 ans. Cependant, la période maximale de transition indiquée dans les différents APE est à nuancer parce que l'analyse des calendriers de libéralisation détaillés montre que la plupart des produits en provenance de l'UE doivent être libéralisés dans une période de moins de 15 ans sachant que pour certains produits, les tarifs douaniers sont réduits de $50 \%$ dès l'entrée en vigueur de l'APE. La période de 25 ans ne concerne que quelques produits considérés comme sensibles et représentant une petite part des importations en provenance de l'UE. D'autres dispositions relatives au TSD comme celles relatives à l'assistance technique, aux mesures de sauvegarde et au traitement particulier des PMA, des Etats enclavés ou insulaires ont été aussi intégrées dans le cadre des APE. Cependant, comme les Etats ACP n'ont cessé de le souligner, les flexibilités dont ils bénéficient sont basées sur une pratique de facto, et non sur une base juridique de jure. Raison pour laquelle ils réclament une réforme de l'Article XXIV du GATT pour prendre en considération les intérêts des pays en développement. Ceci permettrait aux APE d'avoir une base juridique sûre et prévisible au sein du Système Commercial Multilatéral et leur éviterait de subir le même sort que le régime commercial des conventions de Lomé. Il faut aussi souligner que les dispositions relatives au TSD restent généralement des dispositions transitoires raison pour laquelle les Etats ACP et l'UE doivent s'efforcer pour effectivement mettre en œuvre les programmes d'aide pour le commerce. Ceux-ci 
permettront, pendant les périodes transitoires, de répondre aux défis auxquels sont confrontés les Etats ACP.

\section{REFERENCES}

[1] Bilal, S. et F. Rampa. 2006. APE alternatifs et alternatives aux APE: scénarios envisageables pour les futures relations commerciales entre les ACP et l'UE (Rapport ECDPM numéro 11). Maastricht: ECDPM. www.ecdpm.org/pmr11fr

[2] CNUCED, Rapport 2015 sur les Pays les Moins Avancés, (http://unctad.org/fr/PublicationsLibrary/ldc2015_fr.pdf), 20 Avril 2016

[3] OMC, Dispositions relatives au Traitement Spécial et Différencié figurant dans les accords et décisions de l'OMC, Note du secrétariat, WT/COMTD/W/196, 2013, 109 pages.

[4] A. Dicaprio et S.Trommer, Retrouver le principe du Traitement spécial et différencié à travers l'APE du CARIFORUM, Eclairage sur les Négociations, Numéro 8, Volume 6. Juillet-Août 2009, ICTSD

[5] Décision L/4903 du 28 novembre 1979 officiellement appelée «Traitement Différencié et Plus Favorable, Réciprocité et Participation Plus Complète des pays en Voie de Développement». (https://www.wto.org/french/docs_f/legal_f/enabling_f.pdf), 20 Avril 2016

[6] Extrait de l'Article XXIV du GATT

[7] El Hadji A. DIOUF, L'Article XXIV du GATT et l'APE : Arguments Juridiques Pour Soutenir l'Offre Ouest Africaine d'Accès au Marché, ICTSD - Genève, 2009

[8] El Hadji A. DIOUF, L'Article XXIV du GATT et l'offre d'accès au marché dans les APE : Une perspective africaine, Eclairage sur les négociations, Numéro 07 Volume 8. Septembre 2009, ICTSD

[9] Extrait de l'Article 34.2 de l'Accord de Cotonou révisé

[10] Extrait de l'Article 34.2 de l'Accord de Cotonou révisé.

[11] Extrait de l'Article 34.3 de l'Accord de Cotonou révisé.

[12] Dominique Njinkeu, Hugo Cameron (dir), L'aide pour le commerce et le développement, L'Harmattan, 2009 